\title{
Educação Sexual: Estratégias e obstáculos em uma escola pública
}

\author{
Sex Education: Strategies and obstacles in a public school
}

Educación Sexual: Estrategias y obstáculos en una escuela pública

\begin{abstract}
Sheila Maciel da Silva ${ }^{1}$, Jéssica Larissa Pereira dos Santos ${ }^{1}$, Alana Caroline da Silva Rosa ${ }^{1}$, Juliana Pires Rodrigues da Costa ${ }^{1}$, Ruan da Silva Barreto Ferreira ${ }^{1}$, Jefferson Robert Roque de Sousa ${ }^{1}$, Johnata da Cruz Matos ${ }^{1-2 *}$.
\end{abstract}

\section{RESUMO}

Objetivo: identificar os profissionais que trabalham educação sexual, as estratégias utilizadas e os obstáculos encontrados. Método: estudo misto do tipo descritivo, com a participação de sessenta e oito professores. Aplicou-se um questionário e com um roteiro realizaram-se entrevistas semiestruturadas. Os dados foram submetidos à estatística descritiva simples e os relatos à análise temática usada para a construção do discurso do sujeito coletivo. Resultados: a maioria dos professores trabalha o tema principalmente por meio de debate, mas a frequência com que essas atividades acontecem é mínima e insuficiente para promover a prevenção de IST, a gravidez precoce, combater a desigualdade de gênero e permitir que o jovem expresse sua sexualidade e sentimentos de forma saudável. A educação sexual se faz necessária para o desenvolvimento emocional dos adolescentes e para a compreensão do ato sexual enfatizando-se, na abordagem, as consequências. Considerações finais: foi possível identificar os principais aspectos facilitadores e as dificuldades no desenvolvimento do tema em sala de aula. Educação e saúde precisam caminhar juntas, por isso a existência de profissionais da Enfermagem no âmbito escolar, trabalhando com os professores, é avanço na implementação, de forma efetiva, da educação sexual.

Palavras-chave: Sexualidade, Comportamento do Adolescente, Educação em Saúde, Educação Sexual, Enfermagem.

\section{ABSTRACT}

Objective: To identify sex education professionals, the strategies used, and the obstacles encountered. Method: a descriptive mixed study with the participation of sixty-eight teachers. A questionnaire was applied, and a script was conducted semi-structured interviews. Data were submitted to simple descriptive statistics and reports to thematic analysis used to construct the collective subject discourse. Results: Most teachers work on the topic mainly through discussion, but the frequency with which these activities take place is minimal and insufficient to promote STI prevention, early pregnancy, combat gender inequality and allow youth to express their sexuality and feelings in a healthy way. Sex education is necessary for the emotional development of adolescents and for understanding the sexual act, emphasizing the consequences in the approach. Final considerations: it was possible to identify the main facilitating aspects and the difficulties in the development of the theme in the classroom. Education and health need to go together, so the existence of nursing professionals at school, working with teachers, is an advance in the effective implementation of sex education.

Key words: Sexuality, Adolescent Behavior, Health education, Sex Education, Nursing.

${ }^{1}$ Centro Universitário Euro-Americano - UNIEURO. Brasília, Distrito Federal.

2 Universidade de Brasília - UnB. Brasília, Distrito Federal. *E-mail: johnata.matos@unieuro.com.br

SUBMETIDO EM: 9/2019

ACEITO EM: 9/2019

PUBLICADO EM: 10/2019

REAS/EJCH | Vol.Sup.35 | e1653 | DOI: https://doi.org/10.25248/reas.e1653.2019 Página 1 de 10 


\section{RESUMEN}

Objetivo: identificar profesionales de la educación sexual, las estrategias utilizadas y los obstáculos encontrados. Método: estudio descriptivo mixto con la participación de sesenta y ocho docentes. Se aplicó un cuestionario y se realizó un guión de entrevistas semiestructuradas. Los datos se sometieron a estadísticas descriptivas simples e informes al análisis temático utilizado para construir el discurso del sujeto colectivo. Resultados: la mayoría de los docentes trabajan sobre el tema principalmente a través del debate, pero la frecuencia con la que se realizan estas actividades es mínima e insuficiente para promover la prevención de las ITS, el embarazo temprano, combatir la desigualdad de género y permitir que los jóvenes expresen sexualidad y sentimientos de manera saludable. La educación sexual es necesaria para el desarrollo emocional de los adolescentes y para comprender el acto sexual, enfatizando las consecuencias en el enfoque. Consideraciones finales: fue posible identificar los principales aspectos facilitadores y las dificultades en el desarrollo del tema en el aula. La educación y la salud deben ir de la mano, por lo que la existencia de profesionales de enfermería en la escuela, trabajando con maestros, es un avance en la implementación efectiva de la educación sexual.

Palabras clave: Sexualidad, Comportamiento adolescente, Educación en Salud, Educación Sexual, Enfermería.

\section{INTRODUÇÃO}

A adolescência é caracterizada principalmente por transformações biológicas, psicológicas e sociais, nela acontece a puberdade que é uma das fases mais vulneráveis da vida, quando ocorrem diversas maneiras de interação social e, consequentemente, a inclusão do adolescente em outros grupos sociais, além do familiar e, o início da vida sexual (BRASIL, 2010).

Consideram-se os adolescentes um grupo vulnerável ao risco de Infecções Sexualmente Transmissíveis (IST). As IST's são um grande problema de saúde pública e um constante agravo à saúde dos adolescentes, pois aumentam o risco de causar inflamações pélvicas graves, infertilidade, gravidez ectópica, aborto espontâneo, carcinoma de colo uterino, de pênis e ânus, e aumentam o risco de adquirir outra IST como o HIV. Além disso, podem provocar isolamento social e depressão (BRASIL, 2006).

Devido à magnitude desse agravo, interromper a cadeia de transmissão e prevenir novas ocorrências é um desafio à educação e à saúde, o que pode ser feito por meio do estabelecimento de um vínculo entre as escolas e a rede de atenção primária, com ênfase na prevenção por meio de práticas educativas em saúde (GONDIM PS, et al., 2015).

Sabe-se que os adolescentes adquirem informações sobre a prática e a educação sexual por meio da mídia, redes sociais, instituições de ensino, entre outras fontes. Porém, a maioria dessas informações é incompleta. As informações adquiridas pela mídia, sobretudo nas novelas e programas televisionados, a maneira abordada e a temática são constantemente distorcidas da realidade e fantasiosas. Nelas, existem poucas consequências do sexo desprotegido, enfatizando apenas o prazer sexual, mesmo quando ocorre de forma imprudente, fazendo tudo parecer simples e natural (OLIVEIRA LFR, et al., 2015).

Diante da banalização do tema, há uma preocupação apenas com uma possível gravidez indesejada, porém, isso reflete negativamente no conhecimento dos adolescentes sobre o seu próprio corpo, na vivência saudável da sexualidade e na prevenção contra as IST. Os jovens têm dificuldades em interpretar e discernir tais informações. Devido ao comportamento imprudente e por muitas vezes, não conhecer a dimensão das consequências de seus atos, faz-se necessária uma intervenção no contexto das instituições mais próximas, como a família e a escola (QUEIROZ AAFLN, et al., 2016).

Acredita-se que a escola tem o papel fundamental na formação de conceitos, transferência de valores e na promoção da saúde por meio de uma abordagem clara e objetiva na prevenção de IST's e da gravidez não planejada precoce. Logo, a escola é um ambiente favorável e importante para expor os cuidados e 
conhecimentos necessários para uma vida sexual saudável e segura (QUEIROZ AAFLN, et al., 2016). No Brasil, a lei no 60/2009 estabelece o regime de aplicação da educação sexual em meio escolar, onde as escolas devem trabalhar esse tema de grande impacto na saúde.

A qualidade do ensino, conforme o diagnóstico do Plano Nacional de Educação (PNE), só irá se concretizar quando houver a valorização dos profissionais da educação e o reconhecimento da importância do professor no processo de educar. O professor, dentro da instituição de ensino, é essencial na efetivação da informação e conhecimento. Cabe a ele também elaborar métodos para abordar assuntos vinculados à sexualidade utilizando a sua proximidade com o aluno para facilitar o diálogo (SILVA SPC, et al., 2016).

A sexualidade entre pais e filhos é pouco discutida. Nota-se que os pais deixam essa tarefa para os professores que, frente à situação, precisam trabalhar com os alunos o assunto mesmo que não estejam totalmente preparados, pois a sexualidade ainda é um tema cheio de tabus. O diálogo com um profissional preparado, com uma bagagem de informação, contribui positivamente para o entendimento do jovem acerca de hábitos sexuais saudáveis. Conhecimentos específicos, habilidades didáticas, disponibilidade e afinidade para abordar o tema são essenciais. O professor não deve limitar-se apenas ao ensino de sua disciplina, ele deve buscar e explorar outros temas (NOTHAFT SCS, et al., 2014).

Com isso, o objetivo deste estudo foi identificar os profissionais que trabalham educação sexual e quais as principais estratégias utilizadas e obstáculos encontrados.

\section{MÉTODOS}

Estudo descritivo, de abordagem mista. A população escolhida para a realização do estudo é constituída por noventa professores de uma escola pública de ensino médio de Planaltina-DF.

A amostra utilizada foi selecionada por conveniência, ou seja, não probabilística, de acordo com a disponibilidade e interesse dos professores. Realizou-se a pesquisa em uma escola de ensino médio na cidade de Planaltina, DF.

Elencaram-se, como critérios de inclusão, os professores na escola em questão e foram excluídos da pesquisa aqueles que foram remanejados para funções administrativas. Foi possível obter uma amostra final de sessenta e oito participantes configurando-se um nível de significância de $90 \%$ e uma margem de erro amostral de $5 \%$.

Como instrumentos de coleta de dados neste estudo foi ultilizado um questionário estruturado e um roteiro com questões abertas para guiar a entrevista semiestruturada. Todas as entrevistas ocorreram de forma individual, audiogravadas e posteriormente transcritas na íntegra.

No questionário, continha variáveis como sexo, idade, estado civil, filhos, grau de formação, tempo de atuação, abordagem da temática, estratégias e obstáculos. Inicialmente, foi realizado um teste piloto com dois participantes que posteriormente fizeram parte da amostra. O questionário foi aplicado aos demais participantes e foram realizadas dez entrevistas com duração média de 30 minutos.

A análise de dados foi baseada segundo a abordagem quantitativa, em estatística descritiva simples (frequência simples e percentil equivalente) e apresentados em tabelas.

Analisou-se as variáveis no Microsoft Excel 2010 e segundo a abordagem qualitativa, foi realizada uma análise temática usada para a construção do discurso do sujeito coletivo.

As entrevistas foram fundamentadas no Discurso do Sujeito Coletivo (DSC) que é uma técnica de tabulação e organização de dados qualitativos que resolve um dos grandes impasses da pesquisa qualitativa na medida em que permite, por meio de procedimentos sistemáticos e padronizados, agregar depoimentos sem reduzir a quantidade.

Sendo assim, o discurso do sujeito coletivo é um discurso-síntese elaborado com a extração de expressões chaves e ideias centrais que se assemelham entre si representando em um só discurso a temática da pesquisa. 
Foram obedecidos os Critérios da Ética em Pesquisa com Seres Humanos conforme a Resolução $\mathrm{n} .{ }^{\circ}$ 466/2012, do Conselho Nacional de Saúde.

Os participantes da pesquisa foram orientados e assinaram o Termo de Consentimento Livre e Esclarecido. Aprovou-se esta pesquisa pelo Comitê de Ética em Pesquisa do Centro Universitário EuroAmericano - UNIEURO sob o número CAAE: 79439017.0.0000.5056 e parecer n.ํ․ 2.371.609.

\section{RESULTADOS}

Os participantes, em sua maioria, têm idade entre 31 e 40 anos, o que equivale a $38,46 \%$ da amostra. $O$ maior percentual era do sexo masculino, representando $58,82 \%$ dos entrevistados. A maioria tem filhos $(61,19 \%)$ e são casados $(55,22 \%)$. Em relação ao grau de formação, a maior parte $(80,88 \%)$ possui pósgraduação e atua há mais de dez anos na profissão ( $65=67 \%$ dos professores entrevistados). Os dados relacionados à caracterização da amostra estão descritos na Tabela 1. A partir dessa tabela, é observável que algumas questões não foram respondidas por todos os participantes. Mesmo assim, pela relevância do seu conteúdo, serão apresentadas e discutidas (Tabela 1).

Tabela 1 - Dados sociodemográficos coletados dos participantes. Brasília-DF, Brasil, 2018.

\begin{tabular}{lccc}
\hline Características & Especificações & $\mathbf{n}$ & $\%$ \\
\hline Sexo & Feminino & 28 & 41,17 \\
$(\mathrm{n}=68)$ & Masculino & 40 & 58,82 \\
\hline Idade & $20-30$ anos & 07 & 10,76 \\
$(\mathrm{n}=65)$ & $31-40$ anos & 25 & 38,46 \\
& $41-50$ anos & 21 & 32,3 \\
& $>50$ anos & 12 & 18,46 \\
\hline Filhos & Sim & 41 & 61,19 \\
$(\mathrm{n}=67)$ & Não & 26 & 38,8 \\
\hline Estado Civil & Solteiro (a) & 16 & 23,88 \\
$(\mathrm{n}=67)$ & Casado (a) & 37 & 55,22 \\
& Divorciado (a) & 08 & 11,94 \\
& União estável & 05 & 7,46 \\
& Viúvo (a) & 01 & 1,49 \\
\hline Grau de formação & Até - graduação & 13 & 19,11 \\
$(\mathrm{n}=68)$ & Pós-graduação & 55 & 80,88 \\
\hline Tempo de atuação & $0-5$ anos & 11 & 16,41 \\
$(\mathrm{n}=67)$ & $6-10$ anos & 12 & 17,91 \\
& $>10$ anos & 44 & 65,67 \\
\hline
\end{tabular}

Fonte: Silva SM, et al., 2019.

Em relação à experiência teórica em sala de aula, a maioria relata abordar o assunto educação sexual e infecções sexualmente transmissíveis, o que equivale a $63,23 \%$ da amostra. Pouco mais da metade dos pesquisados $(51,47 \%)$ considera apta a discutir a temática e a grande maioria dos professores da amostra acreditam que todas as disciplinas deveriam trabalhar 0 assunto e que a Biologia e a Sociologia, respectivamente, apresentaram um percentual de $33,82 \%$ e $26,47 \%$ como responsáveis por abordar a temática, seguidas por Filosofia, Artes e Química, que adquiriram o mesmo percentual $(16,17 \%)$, como descrito nos dados da (Tabela 2). 
Tabela 2 - Experiência teórica dos professores. Brasília-DF, Brasil, 2018.

\begin{tabular}{|c|c|c|c|c|}
\hline Características & & Especificações & $\mathbf{N}$ & $\%$ \\
\hline Abordagem & Sim & & 43 & 63,23 \\
\hline Sexualidade/IST & & Palestra & 6 & 13,95 \\
\hline \multirow[t]{5}{*}{$(n=68)$} & & Dinâmica & 5 & 11,62 \\
\hline & & Debate & 29 & 67,44 \\
\hline & & Vídeos/filmes & 8 & 18,6 \\
\hline & & Outros & 14 & 32,55 \\
\hline & Não & & 25 & 36,76 \\
\hline Considera-se apto (a) para & Sim & & 35 & 51,47 \\
\hline Trabalhar a temática & Não & & 33 & 48,52 \\
\hline \multirow[t]{6}{*}{$(n=68)$} & & Falta de capacitação & 15 & 45,45 \\
\hline & & Tema divergente da formação acadêmica & 18 & 54,54 \\
\hline & & Falta de recursos materiais & 5 & 15,15 \\
\hline & & Vergonha & 2 & 6,06 \\
\hline & & Fatores religiosos & 1 & 3,03 \\
\hline & & Pressões dos pais de alunos & 4 & 12,12 \\
\hline Disciplinas que devem & & Todas & 42 & 61,76 \\
\hline abordar a temática & & Biologia & 23 & 33,82 \\
\hline \multirow[t]{11}{*}{$(n=68)$} & & Educação física & 8 & 11,76 \\
\hline & & Português & 2 & 2,94 \\
\hline & & Geografia & 2 & 2,49 \\
\hline & & Matemática & 0 & 0 \\
\hline & & Física & 0 & 0 \\
\hline & & Língua Estrangeira & 1 & 1,47 \\
\hline & & História & 6 & 8,82 \\
\hline & & Filosofia & 11 & 16,17 \\
\hline & & Sociologia & 18 & 26,47 \\
\hline & & Química & 2 & 16,17 \\
\hline & & Artes & 11 & 16,17 \\
\hline
\end{tabular}

Fonte: Silva SM, et al., 2019.

Quanto à experiência prática, $33,82 \%$ dos entrevistados nunca realizam atividades voltadas ao tema. $\mathrm{A}$ porcentagem que realiza o faz com pouca frequência (uma vez ao ano, equivalente a 30,88\%) e o menor percentual $(2,94 \%)$ faz com a frequência de uma vez por semana.

Em relação à escolha do tema, 50\% (representando o maior percentual) apontam o professor como responsável pela escolha.

Quanto à realização de trabalhos de sensibilização com os pais, a maioria $(89,06 \%)$ relata nunca fazer. Sobre a presença de profissional de saúde na escola, 33,33\% marcaram nunca existir, chamando a atenção, pois alguns professores assinalaram a existência de profissionais da saúde na escola para debater temas relacionados à educação sexual e IST com alguma frequência, enquanto outros assinalaram não existir, conforme exposto na (Tabela 3). 
Tabela 3 - Experiência prática dos professores. Brasília-DF, Brasil, 2018.

\begin{tabular}{lccc}
\hline Características & Especificações & $\mathbf{N}$ & $\%$ \\
\hline Frequência de atividades & Nunca & 23 & 33,82 \\
Ligadas ao tema na & 1vez/ano & 21 & 30,88 \\
Disciplina que leciona & 1 vez/mês & 13 & 19,11 \\
( $\mathrm{n}=68)$ & 1vez/ semana & 2 & 2,94 \\
& Quando o aluno questiona & 9 & 13,23 \\
\hline Responsável pela escolha & Todos & 24 & 38,7 \\
do tema & Professor & 31 & 50 \\
(n=62) & Direção da escola & 4 & 6,45 \\
& Aluno & 7 & 11,29 \\
\hline Trabalho de sensibilização & Sim & 7 & 10,93 \\
com os pais & Não & 57 & 89,06 \\
( $n=64)$ & & & \\
\hline Presença de profissional de & Às vezes & 15 & 23,8 \\
Saúde na escola & Nunca & 21 & 33,33 \\
(n=63) & Raramente & 18 & 28,57 \\
\end{tabular}

Fonte: Silva SM, et al., 2019.

A partir dos relatos dos participantes foi possível inferir que os docentes percebem a relevância de trabalhar a sexualidade no âmbito escolar e levantam, como justificativas para isso, o risco de sofrimento psicológico, de uma gravidez sem planejamento e de aquisição de infecções sexualmente transmissíveis.

"Através de debates e conversas em grupo, podemos evitar o sofrimento psicológico que uma gravidez indesejada ou uma IST pode trazer para o adolescente na medida que o educando vai se apropriando de sua identidade. Por isso, trabalhar educação sexual no ambiente escolar é de grande relevância, é essencial que os professores de todas as áreas trabalhem essa temática, pois é com o conhecimento correto do assunto que se evita problemas futuros". DSC 1

Observou-se que os adolescentes têm acesso a poucas informações sobre sexualidade no ambiente familiar. Sendo assim, a maior parte do seu conhecimento é adquirida por meio das mídias que fornecem, na maioria das vezes, um conteúdo que não condiz com a realidade enfatizando um comportamento imprudente e dificultando a formação de conceitos e o comportamento sexual saudável.

"É possível notar que a maior dificuldade é ter a orientação dentro do próprio lar, seja por motivo religioso ou por falta de conhecimentos dos pais. Nesse caso, as informações que esses adolescentes têm vêm por meio de filmes ou até mesmo rodas de conversas com amigos, que muitas vezes, não possuem as informações corretas, podendo ser perigoso pois a informação errada os levará a agir de forma errada e assim obterem graves consequências". DCS 2

A educação sexual é necessária para o desenvolvimento emocional agregando valores essenciais para o relacionamento dos adolescentes e para a compreensão do ato sexual em toda sua plenitude enfatizandose, na abordagem, as consequências. Os profissionais de educação/saúde devem trabalhar a educação sexual de forma que os alunos compreendam o valor do ato sexual e os seus riscos, além de contribuir para o autocuidado em relação à sexualidade dos adolescentes. 


\section{DISCUSSÃO}

Verificou-se neste estudo o predomínio de profissionais com idade superior aos 31 anos, sendo os maiores percentuais entre 31 e 40 anos e 41 e 50 anos, correspondendo respectivamente a $38,46 \%$ e $32,3 \%$ dos pesquisados, o que se confirma por meio dos dados nacionais do censo da educação básica onde a faixa de 30 a 49 é a que prevalece em $70,5 \%$ dos profissionais atuantes. Este dado também explica o tempo de atuação na docência maior onde $67 \%$ dos pesquisados possuem tempo de atuação superior a dez anos. Neste estudo, existe uma predominância de $80,88 \%$ de profissionais com pós-graduação. Esse achado é compatível com os dados nacionais onde é crescente o número de docentes em busca de especialização e com curso superior (BRASIL, 2013a).

Os profissionais da saúde e educação são essenciais na composição de um país. No Brasil, correspondem, em conjunto, a cerca de $20 \%$ das ocupações profissionais. Dentre os docentes do ensino médio, observa-se que a maior parte dos profissionais é do sexo feminino, correspondendo $67 \%$ da amostra (BRASIL, 2013b).

Liga-se diretamente a existência da qualidade de ensino ao reconhecimento e valorização da classe docente, sendo necessário investimento por meio de melhoria dos salários, da qualidade no ambiente e de condições de trabalho, incentivo à carreira e à formação continuada, onde o avanço da educação básica será consequência do preparo dos professores sucedendo justamente das possibilidades disponibilizadas a eles (SILVA SPC, et al., 2016).

Apesar da maior parte dos entrevistados trabalhar o tema educação sexual e infecções sexualmente transmissíveis, pouco mais da metade se considera apta a realizar essa intervenção. Entre os principais motivos assinalados, destaca-se o tema ser divergente da área de formação e a falta de capacitação. Vale ressaltar a responsabilidade da escola em assegurar os direitos do aluno como o acesso à informação e à saúde, componente apontado como necessário à formação de pessoas responsáveis e cientes da sua capacidade (BANDEIRA J, et al., 2016).

Existem várias políticas governamentais direcionadas a assegurar os direitos sexuais e reprodutivos no setor da educação. Nesse contexto, a escola é vista como um local para a promoção do reconhecimento da diversidade de expressão sexual. Porém, realizar tal função não é algo simples, pois as pessoas que atuam na área têm vivenciado obstáculos na efetivação do que propõem os documentos oficiais. A sexualidade é algo complexo que abrange questões culturais, históricas, biológicas e políticas que fazem parte dos valores e experiências das pessoas que estão envolvidas nesse contexto (GESSER M, et al., 2015).

As perspectivas em relação a crenças, valores e costumes culturais de homens e mulheres influenciam e sustentam relações de gênero desiguais. Historicamente, tais relações têm beneficiado o homem, porém, esses conceitos continuam sendo alimentados tanto por homens, quanto por mulheres. A desvalorização da mulher ainda é algo comum na atualidade, pois há reprodução de valores arcaicos sobre este universo feminino, que demonstram um ponto de vista depreciativo acerca da mulher e sua sexualidade (MADUREIRA AFA e BRANCO AU, 2015).

Outra questão envolvendo a figura feminina é a gravidez na adolescência, apontada como um problema social e um transtorno à saúde pública que desencadeiam situações como: o mau desempenho e o abandono escolar; perigos no curso da gravidez; falta de comunicação da gravidez por medo de represálias, por vergonha ou receio de como a notícia será recebida acabando por não realizar o pré-natal ou por começá-lo de forma tardia, além da discriminação social e do desentendimento familiar (MADEIRA BM, 2015).

As IST's são também um problema grave de saúde e um dano à vida dos adolescentes escolares. O Brasil é apontado como um dos países com grande número de notificações de Aids, onde a população jovem é a mais acometida.

Além disso, as informações epidemiológicas sobre as IST's são limitadas por conta das subnotificações, o que dificulta a tomada de ações para a prevenção e o controle em maior magnitude que possam reduzir os índices de contaminação (SILVA SPC, et al., 2016). 
O acesso à informação de qualidade é uma ferramenta importante na prevenção das IST's entre jovens escolares. É preciso destacar a falta de capacitação na área de IST e sexualidade observada em outros estudos, mostrando que mesmo com a existência de algumas políticas no ambiente escolar, essas ainda não alcançaram os objetivos esperados. Salienta-se que, em outros estudos, grande parte dos professores, mesmo tendo um bom grau de escolaridade e considerar importante falar sobre esse assunto, ainda tem dificuldade para discutir o tema sexualidade. Outros autores descrevem também que os docentes se consideram despreparados (SILVA SPC, et al., 2016).

Chama-se a atenção para um dado neste estudo: mesmo a maior parte dos professores assinalando que trabalha a temática, a frequência da abordagem é insatisfatória. Apenas uma vez ao ano é o maior percentual, muito diferente do que se espera, já que a escola é um lócus oportuno para a expansão de técnicas de cuidado onde os professores poderiam favorecer, de forma mútua e dinâmica, um espaço dedicado para tirar e esclarecer dúvidas sobre a sexualidade. Esse tipo de ação contribuiria para a redução de danos relacionados à vida sexual e reprodutiva precoce dos alunos (MADUREIRA AFA e BRANCO AU, 2015).

Além da pouca frequência na abordagem do tema sexualidade, o educador que não tem conhecimento necessário para abordar o tema no ambiente escolar o faz somente quando acontece um questionamento por parte do aluno sobre o assunto, sendo trabalhado assim, apenas esporadicamente e não de forma contínua. Dessa forma, assuntos como gravidez durante a adolescência e infecções sexualmente transmissíveis deixam de ser debatidos em conjunto com os alunos e não recebem a devida importância (BANDEIRA J, et al., 2016).

Há várias dificuldades para discorrer o tema pelos educadores, entre os motivos estão a falta de formação específica e a carga horária intensa e exaustiva, uma vez que boa parte leciona em mais de uma escola, além da carência de materiais didáticos, obstáculos na adesão dos demais professores em abordar o assunto interdisciplinarmente, falta de entusiasmo ou preocupação em discutir o tema e não encontrar um modo de orientar. Frente a essa série de problemas em trabalhar a educação sexual no ambiente escolar, ela acaba acontecendo de modo rápido e momentâneo (BANDEIRA J, et al., 2016).

A sexualidade é um tema abrangente e complexo que não deve se restringir à área das Ciências Biológicas. Neste estudo, a maioria dos pesquisados assinalou que todas as disciplinas deveriam abordar o tema, porém, uma boa parte aponta a Biologia, como uma das mais adequadas para a abordagem, diferente do que seria adequado, pois a sexualidade é um tema extenso que envolve muitas questões e não pode estar limitada a discursos formais e às matérias de Ciências Biológicas com foco apenas na reprodução humana e no aparelho reprodutivo (SILVA SPC, et al., 2016).

Inseriu-se a educação sexual no Brasil, no currículo escolar, na década de 1960. No início, a abordagem da sexualidade era pautada na prevenção da gravidez na adolescência e nas infecções sexualmente transmissíveis. A partir de 1996, houve uma mudança da educação sexual no ambiente escolar por meio da implantação dos Parâmetros Curriculares Nacionais (PCN), com o propósito de uma abordagem mais ampla envolvendo todos os aspectos da vida sexual e contemplando o homem com uma visão holística (PINHEIRO AS, et al., 2017).

Por meio das Diretrizes Curriculares Nacionais, a educação sexual deve ser abordada de forma transversal, ou seja, sendo discutida nos momentos em que surgirem situações relacionadas à temática. Para o desenvolvimento dessa abordagem, é necessário que o docente tenha um conhecimento que vai além da sua área de formação possibilitando a discussão minuciosa sobre a sexualidade. $O$ docente não necessita de uma especialização em educação sexual, mas é necessário que ele possua fundamentos teóricos para a reflexão do tema (MARCON AN, et al., 2016).

É fundamental o papel da família no desenvolvimento dos adolescentes, por isso é de suma importância trabalhar o amadurecimento da sexualidade, porém o diálogo entre pais e filhos é limitado, isso prejudica a compreensão e reflete, de forma negativa, no comportamento sexual (SOUZA V, et al., 2017).

Durante a adolescência, há uma busca pela identidade que proporciona mudanças fundamentais para o desenvolvimento do indivíduo. Nessa fase, o adolescente carece da construção de relações afetivas. Para o 
desenvolvimento dessas relações, eles necessitam do apoio, afeto e proteção da família (KAMALA BA, et al., 2017). A sexualidade, nessa etapa de transformações corporais e hormonais, é envolvida por questionamentos e descobertas focalizando-se no prazer vivências (GOLDMAN JG e GRIMBEEK P, 2016).

$\mathrm{O}$ adolescente conduz a sua vida sexual de acordo com o seu conhecimento sobre valores e normas sociais. Assim, a experiência do sujeito depende desse conhecimento, contribuindo para uma melhor relação com o seu corpo e os seus relacionamentos (CASTRO F, et al., 2018).

Diante desse cenário, a educação sexual precisa explanar o conceito de sexualidade abordando os aspectos emocional e biológico, o respeito, a convivência e os padrões e as normas sociais (DE CASTRO F, et al., 2018).

Por isso, é necessário discutir a sexualidade não somente por meio das ciências biológicas, mas por todas as disciplinas, bem como envolver toda a equipe pedagógica em parceria com a família e os profissionais de saúde atuantes naquela área de abrangência (SILVA SPC, et al., 2016).

\section{CONCLUSÃO}

A maioria dos professores trabalha o tema por meio de debate, mas a frequência com que essas atividades acontecem é mínima, insuficiente para promover a prevenção de IST, a gravidez precoce, combater a desigualdade de gênero e permitir que o jovem expresse sua sexualidade e sentimentos de forma saudável, além de entender a plenitude do ato sexual e suas consequências. Logo, a educação e saúde precisam caminhar juntas. A existência de profissionais da área de Enfermagem no âmbito escolar, trabalhando em conjunto com os professores, seria um avanço na implementação, de forma efetiva, da educação sexual.

\section{REFERÊNCIAS}

1. BELÉM MB, ALENCAR G, NUNES JFC, et al. Sex knowledge and practices of teen male: health impact. R Enferm Cent O Min, 2014 May/Aug; 4(2):1146-62.

2. BANDEIRA J, Perception of educators on sexual guidance at school: a floor they never treaded. J Nurs UFPE on line, 2016 Mar; 10(3):1102-8.

3. BRASIL. Lei $n^{\circ} 60$ de 6 de agosto de 2009 (BR). Estabelece o regime de aplicação da educação sexual em meio escolar. Diário Oficial da União. 2009.

4. BRASIL. Ministério da Educação. Instituto Nacional de Estudos e Pesquisas Educacionais Anísio Teixeira. Diretoria de Estatísticas Educacionais. Censo da educação básica: 2012 - resumo técnico. Brasília: INEP; 2013a.

5. BRASIL. Ministério da Educação. Secretaria de Educação Básica Secretaria de Educação Continuada, Alfabetização, Diversidade e Inclusão Conselho Nacional de Educação. Diretrizes Curriculares Nacionais da Educação Básica. Brasília: Ministério da Educação; 2013b.

6. BRASIL. Ministério da Saúde. Secretaria de Atenção em Saúde, Departamento de Ações Programáticas Estratégicas. Diretrizes nacionais para a atenção integral à saúde de adolescentes e jovens na promoção, proteção e recuperação da saúde. Brasília: Ministério da Saúde, 2010.

7. BRASIL. Ministério da Saúde. Secretaria de Vigilância em Saúde. Programa Nacional de DST e Aids. Manual de Controle das Doenças Sexualmente Transmissíveis. 4th ed. Brasília: Ministério da Saúde; 2006.

8. CASTRO F, ROJAS-MARTÍNEZ R, VILLALOBOS-HERNÁNDEZ, et al. Sexual and reproductive health outcomes are positively associated with comprehensive sexual education exposure in Mexican high-school students. Plos One, 2018 Mar; 13(3): e0193780.

9. GESSER M. Teaching and concepts of sexuality in fundamental education. Psicol Soc, $2015 \mathrm{Sept} / \mathrm{Dec}$; 27(3): 558-68, 2015.

10. GOLDMAN JG, GRIMBEEK P. What do preservice teachers want to learn about puberty and sexuality education? An Australian perspective. Pastor Care Educ, 2016 Dec; 34(4): 189-201.

11. GONDIM PS. Accessibility of adolescents to sources of information on sexual and reproductive health. Revista Brasileira de Crescimento e Desenvolvimento Humano, 2015; 25(1): 50-3.

12. KAMALA BA. Evaluation of the Families Matter! Program in Tanzania: An Intervention to Promote Effective Parent-Child Communication About Sex, Sexuality, and Sexual Risk Reduction. AIDS Educ Prevent, 2017 Apr; 29(2): 105-20. 
13. MACHADO GM, PIRES RG. Gender identity and implications on sexuality in teacher perspective of Education Physical. Motrivivência, 2016 Sept; 28(8): 360-75.

14. MADEIRA BM. Promoção da saúde e prevenção de gravidez para adolescentes do ensino fundamental e médio da Escola Coronel Silvino Pereira, no município de Coronel Fabriciano - Minas Gerais [monograph]. Ipatinga: UFMG; 2015.

15. MADUREIRA AFA, BRANCO AU. Gender, sexuality and diversity in schools from teachers' perspective. Temas psicol, 2015 Sept; 23(3): 577-91.

16. MARCON AN. Public policies related to sexual diversity in school. Psicologia Escolar e Educacional, 2016 May/Aug; 20(2): 291-301.

17. NOTHAFT SCS. Educators' perspective on adolescent sexuality: possible education practices. Revista Mineira de Enfermagem, 2014; 18(2): 284-9.

18. OLIVEIRA LFR. Adesão de adolescentes à camisinha masculina. J Res Fundam Care online, 2015 Jan/Mar; 7(1): 1765-73.

19. PINHEIRO AS. The family health strategy and the school in sex education: an intersectoral perspective. Trab educ saúde, 2017 Sept/Dec; 15(3): 803-22.

20. QUEIROZ AAFLN. Sex education for adolescents by teachers from a community education center. J Res Fundam Care online, 2016 Oct/Dec; 8(4): 5120-5.

21. SILVA SPCI. Discussing sexuality/sti in the school context: public school teacher's practices. J Nurs UFPE online, 2016 Nov; 10(5): 4295-303.

22. SOUZA V. Knowledge, experiences and beliefs in the sexual field: a study of 1 and 2 year high school students from different socioeconomic backgrounds. Revista Mineira de Enfermagem, 2017; 21 : e-991. 